\title{
On the modified $q$-Euler polynomials with weight
}

\author{
Seog-Hoon Rim ${ }^{1}$, Jin-Woo Park*, Jongkyum Kwon² and Sung-Soo Pyo ${ }^{1}$
}

"Correspondence:

a0417001@knu.ac.kr

${ }^{1}$ Department of Mathematics

Education, Kyungpook National

University, Taegu, 702-701, Republic of Korea

Full list of author information is

available at the end of the article

\section{Abstract}

In this paper, we construct a new q-extension of Euler numbers and polynomials with weight related to fermionic $p$-adic $q$-integral on $\mathbb{Z}_{p}$ and give new explicit formulas related to these numbers and polynomials.

Keywords: modified $q$-Euler polynomials; modified $q$-Euler polynomials with weight; fermionic $p$-adic $q$-integral on $\mathbb{Z}_{p}$

Throughout this paper $\mathbb{Z}_{p}, \mathbb{Q}_{p}$ and $\mathbb{C}_{p}$ will respectively denote the ring of $p$-adic integers, the field of $p$-adic rational numbers and the completion of algebraic closure of $\mathbb{Q}_{p}$. Let $v_{p}$ be the normalized exponential valuation of $\mathbb{C}_{p}$ with $|p|_{p}=p^{-v_{p}(p)}=\frac{1}{p}$.

In this paper, we assume that $q \in \mathbb{C}_{p}$ with $|1-q|_{p}<p^{-\frac{1}{p-1}}$ so that $q^{x}=\exp (x \log q)$ for $x \in \mathbb{Z}_{p}$. The $q$-number of $x$ is denoted by $[x]_{q}=\frac{1-q^{x}}{1-q}$. Note that $\lim _{q \rightarrow 1}[x]_{q}=x$. Let $d$ be a fixed integer bigger than 0 , and let $p$ be a fixed prime number and $(d, p)=1$. We set

$$
\begin{aligned}
& X_{d}=\lim _{\overleftarrow{N}} \mathbb{Z} / d p^{N} \mathbb{Z}, \quad X^{*}=\bigcup_{\substack{0<a<d p \\
(a, p)=1}}\left(a+d p \mathbb{Z}_{p}\right), \\
& a+d p^{N} \mathbb{Z}_{p}=\left\{x \in X \mid x \equiv a\left(\bmod d p^{N}\right)\right\},
\end{aligned}
$$

where $a \in \mathbb{Z}$ lies in $0 \leq a<d p^{N}$ (see [1-22]).

Let $C\left(\mathbb{Z}_{p}\right)$ be the space of continuous functions on $\mathbb{Z}_{p}$. For $f \in C\left(\mathbb{Z}_{p}\right)$, the fermionic $p$ adic q-integral on $\mathbb{Z}_{p}$ is defined by Kim as

$$
I_{q}(f)=\int_{\mathbb{Z}_{p}} f(x) d \mu_{-q}(x)=\lim _{N \rightarrow \infty} \frac{1}{\left[p^{N}\right]_{q}} \sum_{x=0}^{p^{N}-1} f(x)(-q)^{x} \quad \text { (see [8-22]). }
$$

As is well known, Euler polynomials are defined by the generating function to be

$$
\frac{2}{e^{t}+1} e^{x t}=e^{E(x) t}=\sum_{n=0}^{\infty} E_{n}(x) \frac{t^{n}}{n !} \quad(\text { see }[11-13,15,20-22])
$$

with the usual convention about replacing $E^{n}(x)$ by $E_{n}(x)$. In the special case, $x=0$, $E_{n}(0)=E_{n}$ are called the $n$th Euler numbers.

@2013 Rim et al.; licensee Springer. This is an Open Access article distributed under the terms of the Creative Commons Attribution License (http://creativecommons.org/licenses/by/2.0), which permits unrestricted use, distribution, and reproduction in any medium, provided the original work is properly cited. 
In [13, 20, 23], Kim defined the q-Euler numbers as follows:

$$
E_{0, q}=1, \quad q(q E+1)^{n}+E_{n, q}= \begin{cases}{[2]_{q},} & \text { if } n=0, \\ 0, & \text { if } n \neq 0\end{cases}
$$

with the usual convection of replacing $E^{n}$ by $E_{n, q}$. From (1), we also derive

$$
E_{n, q}=\frac{[2]_{q}}{(1-q)^{n}} \sum_{l=0}^{n}\left(\begin{array}{l}
n \\
l
\end{array}\right) \frac{(-1)^{l}}{1+q^{l+1}} \quad(\text { see }[20,23])
$$

By using an invariant $p$-adic $q$-integral on $\mathbb{Z}_{p}$, a $q$-extension of ordinary Euler polynomials, called $q$-Euler polynomials, is considered and investigated by Kim $[14,15,18]$. For $x \in \mathbb{Z}_{p}, q$-Euler polynomials are defined as follows:

$$
E_{n, q}(x)=\int_{\mathbb{Z}_{p}}[x+y]_{q}^{n} d \mu_{-q}(y)
$$

By (2), the following relation holds:

$$
E_{n, q}(x)=\sum_{k=0}^{n}\left(\begin{array}{l}
n \\
k
\end{array}\right)[x]_{q}^{n-k} q^{k x} E_{k, q}
$$

Recently, Kim considered the modified $q$-Euler polynomials which are slightly different from Kim's $q$-Euler polynomials as follows:

$$
\epsilon_{n, q}(x)=\int_{\mathbb{Z}_{p}} q^{-x}[x+y]_{q}^{n} d \mu_{-q}(y) \quad \text { for } n \in \mathbb{N},
$$

and he showed that

$$
\epsilon_{n, q}(x)=\frac{[2]_{q}}{(1-q)^{n}} \sum_{l=0}^{n}\left(\begin{array}{l}
n \\
l
\end{array}\right) \frac{q^{x l}}{1+q^{l}}
$$

(see [22]). In the special case, $x=0, \epsilon_{n, q}(0)=\epsilon_{n, q}$ are called the $n$th modified $q$-Euler numbers, and it is showed that

$$
\epsilon_{n, q}=\frac{[2]_{q}}{(1-q)^{n}} \sum_{l=0}^{n}\left(\begin{array}{l}
n \\
l
\end{array}\right) \frac{1}{1+q^{l}} .
$$

And in [24], authors defined modified q-Euler polynomials with weight $\alpha \epsilon_{n, q}^{(\alpha)}(x)$ as follows:

$$
\epsilon_{n, q}^{(\alpha)}(x)=\int_{\mathbb{Z}_{p}} q^{-x}[x+y]_{q^{\alpha}}^{n} d \mu_{-q^{\alpha}}(y)
$$

and proved that

$$
\epsilon_{n, q}^{(\alpha)}(x)=\frac{[2]_{q}}{\left(1-q^{\alpha}\right)^{n}} \sum_{l=0}^{n}\left(\begin{array}{l}
n \\
l
\end{array}\right)(-1)^{l} \frac{q^{\alpha l}}{1+q^{\alpha l}} .
$$


In the special case, $x=0, \epsilon_{n, q}^{(\alpha)}(0)=\epsilon_{n, q}^{(\alpha)}$ are called the $n$th modified $q$-Euler numbers with weight $\alpha$, and it is showed that

$$
\begin{aligned}
\epsilon_{n, q}^{(\alpha)} & =\frac{[2]_{q}}{\left(1-q^{\alpha}\right)^{n}} \sum_{l=0}^{n}\left(\begin{array}{l}
n \\
l
\end{array}\right)(-1)^{l} q^{\alpha l} \frac{1}{1+q^{\alpha l}} \\
& =[2]_{q} \sum_{m=0}^{\infty}(-1)^{m}[m+x]_{q^{\alpha}}^{n} .
\end{aligned}
$$

In this paper, we construct a new $q$-extension of Euler numbers and polynomials with weight related to fermionic $p$-adic $q$-integral on $\mathbb{Z}_{p}$ and give new explicit formulas related to these numbers and polynomials.

\section{A new approach of modified $q$-Euler polynomials}

Let us consider the following modified q-Euler numbers:

$$
\begin{aligned}
\tilde{\epsilon}_{n, q}(x) & =\int_{\mathbb{Z}_{p}} q^{-y}\left(x+[y]_{q}\right)^{n} d \mu_{-q}(y) \\
& =\sum_{l=0}^{n}\left(\begin{array}{l}
n \\
l
\end{array}\right) x^{n-l} \epsilon_{l, q}=\sum_{l=0}^{n} \sum_{k=0}^{l}\left(\begin{array}{l}
n \\
l
\end{array}\right)\left(\begin{array}{l}
l \\
k
\end{array}\right) \frac{[2]_{q}}{(1-q)^{l}} \frac{x^{n-l}}{1+q^{k}},
\end{aligned}
$$

where

$$
\tilde{\epsilon}_{n, q}(0)=\epsilon_{n, q}=\frac{[2]_{q}}{(1-q)^{n}} \sum_{l=0}^{n}\left(\begin{array}{l}
n \\
l
\end{array}\right) \frac{1}{1+q^{l}} .
$$

Thus, by (7),

$$
(1-q)^{n} \epsilon_{n, q}=[2]_{q} \sum_{l=0}^{n}\left(\begin{array}{l}
n \\
l
\end{array}\right) \frac{1}{1+q^{l}} .
$$

Consider the equation

$$
\begin{aligned}
\sum_{n=0}^{\infty}(1-q)^{n} \epsilon_{n, q} \frac{t^{n}}{n !} & =[2]_{q} \sum_{n=0}^{\infty} \sum_{l=0}^{n}\left(\begin{array}{l}
n \\
l
\end{array}\right) \frac{1}{1+q^{l}} \frac{t^{n}}{n !}=[2]_{q}\left(\sum_{m=0}^{\infty} \frac{t^{m}}{m !}\right)\left(\sum_{l=0}^{\infty} \frac{1}{1+q^{l}} \frac{t^{l}}{l !}\right) \\
& =[2]_{q} e^{t}\left(\sum_{l=0}^{\infty} \frac{1}{1+q^{l}} \frac{t^{l}}{l !}\right) .
\end{aligned}
$$

Since

$$
\begin{aligned}
e^{(1-q) x t} \sum_{n=0}^{\infty}(1-q)^{n} \epsilon_{n, q} \frac{t^{n}}{n !} & =\left(\sum_{l=0}^{\infty} \frac{(1-q)^{l} x^{l} t^{l}}{l !}\right)\left(\sum_{n=0}^{\infty}(1-q)^{n} \epsilon_{n, q} \frac{t^{n}}{n !}\right) \\
& =\sum_{m=0}^{\infty}(1-q)^{m} \sum_{n=0}^{m}\left(\begin{array}{c}
m \\
n
\end{array}\right) \epsilon_{n, q} x^{m-n} \frac{t^{m}}{m !} \\
& =\sum_{m=0}^{\infty}(1-q)^{m} \tilde{\epsilon}_{m, q}(x) \frac{t^{m}}{m !}
\end{aligned}
$$


and

$$
\begin{aligned}
e^{(1-q) x t}[2]_{q} e^{t}\left(\sum_{l=0}^{\infty} \frac{1}{1+q^{l}} \frac{t^{l}}{l !}\right) & =[2]_{q} e^{((1-q) x+1) t}\left(\sum_{l=0}^{\infty} \frac{1}{1+q^{l}} \frac{t^{l}}{l !}\right) \\
& =[2]_{q}\left(\sum_{m=0}^{\infty}((1-q) x+1)^{m} \frac{t^{m}}{m !}\right)\left(\sum_{l=0}^{\infty} \frac{1}{1+q^{l}} \frac{t^{l}}{l !}\right) \\
& =[2]_{q} \sum_{n=0}^{\infty} \sum_{l=0}^{n}\left(\begin{array}{l}
n \\
l
\end{array}\right) \frac{((1-q) x+1)^{n-l}}{1+q^{l}} \frac{t^{n}}{n !},
\end{aligned}
$$

by (8) and (9), we get

$$
\begin{aligned}
(1-q)^{n} \tilde{\epsilon}_{n, q}(x) & =[2]_{q} \sum_{l=0}^{n}\left(\begin{array}{l}
n \\
l
\end{array}\right) \frac{((1-q) x+1)^{n-l}}{1+q^{l}} \\
& =[2]_{q} \sum_{l=0}^{n}\left(\begin{array}{l}
n \\
l
\end{array}\right) \frac{1}{1+q^{l}} \sum_{j=0}^{n-l}\left(\begin{array}{c}
n-l \\
j
\end{array}\right)(1-q)^{j} x^{j} .
\end{aligned}
$$

Thus, we have the following result.

Theorem 1.1 For $n \geq 1$,

$$
\begin{aligned}
\tilde{\epsilon}_{n, q}(x) & =\frac{[2]_{q}}{(1-q)^{n}} \sum_{l=0}^{n}\left(\begin{array}{c}
n \\
l
\end{array}\right) \frac{((1-q) x+1)^{n-l}}{1+q^{l}} \\
& =\frac{[2]_{q}}{(1-q)^{n}} \sum_{l=0}^{n} \sum_{j=0}^{n-l}\left(\begin{array}{c}
n \\
l
\end{array}\right)\left(\begin{array}{c}
n-l \\
j
\end{array}\right) \frac{(1-q)^{j}}{1+q^{l}} x^{j} .
\end{aligned}
$$

\section{A new approach of $q$-Euler polynomials with weight $\alpha$}

Let us consider the following modified q-Euler polynomials with weight $\alpha$ :

$$
\begin{aligned}
\tilde{\epsilon}_{n, q}^{(\alpha)}(x) & =\int_{\mathbb{Z}_{p}} q^{-y}\left(x+[y]_{q^{\alpha}}\right)^{n} d \mu_{-q^{\alpha}}(y) \\
& =\sum_{l=0}^{n}\left(\begin{array}{l}
n \\
k
\end{array}\right) x^{n-l} \epsilon_{k, q}^{(\alpha)}=\sum_{k=0}^{n} \sum_{l=0}^{k}\left(\begin{array}{l}
n \\
k
\end{array}\right)\left(\begin{array}{l}
k \\
l
\end{array}\right) \frac{[2]_{q^{\alpha}}}{(1-q)^{n}} \frac{(-1)^{l}}{1+q^{\alpha+l}} x^{n-k},
\end{aligned}
$$

where

$$
\tilde{\epsilon}_{n, q}^{(\alpha)}(0)=\epsilon_{n, q}^{(\alpha)}=\frac{[2]_{q}}{\left(1-q^{\alpha}\right)^{n}} \sum_{l=0}^{n}\left(\begin{array}{l}
n \\
l
\end{array}\right) \frac{(-1)^{l} q^{\alpha l}}{1+q^{\alpha l}} .
$$

Thus, by (10), we have

$$
\left(1-q^{\alpha}\right)^{n} \epsilon_{n, q}^{(\alpha)}=[2]_{q} \sum_{l=0}^{n}\left(\begin{array}{l}
n \\
l
\end{array}\right) \frac{(-1)^{l} q^{\alpha l}}{1+q^{\alpha l}} .
$$


Consider the equation

$$
\begin{aligned}
\sum_{n=0}^{\infty}\left(1-q^{\alpha}\right)^{n} \epsilon_{n, q}^{(\alpha)} \frac{t^{n}}{n !} & =[2]_{q} \sum_{n=0}^{\infty} \sum_{l=0}^{n}\left(\begin{array}{l}
n \\
l
\end{array}\right) \frac{(-1)^{l} q^{\alpha l}}{1+q^{\alpha l}} \frac{t^{n}}{n !}=[2]_{q}\left(\sum_{m=0}^{\infty} \frac{t^{m}}{m !}\right)\left(\sum_{l=0}^{\infty} \frac{(-1)^{l} q^{\alpha l}}{1+q^{\alpha l}} \frac{t^{l}}{l !}\right) \\
& =[2]_{q} e^{t}\left(\sum_{l=0}^{\infty} \frac{\left(-q^{\alpha}\right)^{l}}{1+q^{\alpha l}} \frac{t^{l}}{l !}\right) .
\end{aligned}
$$

Since

$$
\begin{aligned}
e^{\left(1-q^{\alpha}\right) x t} \sum_{n=0}^{\infty}\left(1-q^{\alpha}\right)^{n} \epsilon_{n, q}^{(\alpha)} \frac{t^{n}}{n !} & =\left(\sum_{l=0}^{\infty} \frac{\left(1-q^{\alpha}\right)^{l} x^{l} t^{l}}{l !}\right)\left(\sum_{n=0}^{\infty}\left(1-q^{\alpha}\right)^{n} \epsilon_{n, q}^{(\alpha)} \frac{t^{n}}{n !}\right) \\
& =\sum_{m=0}^{\infty}\left(1-q^{\alpha}\right)^{m} \sum_{n=0}^{m}\left(\begin{array}{c}
m \\
n
\end{array}\right) \epsilon_{n, q}^{(\alpha)} x^{m-n} \frac{t^{m}}{m !} \\
& =\sum_{m=0}^{\infty}\left(1-q^{\alpha}\right)^{m} \tilde{\epsilon}_{m, q}^{(\alpha)}(x) \frac{t^{m}}{m !}
\end{aligned}
$$

and

$$
\begin{aligned}
e^{\left(1-q^{\alpha}\right) x t}[2]_{q} e^{t}\left(\sum_{l=0}^{\infty} \frac{\left(-q^{\alpha}\right)^{l}}{1+q^{\alpha l}} \frac{t^{l}}{l !}\right) & =[2]_{q} e^{\left(\left(1-q^{\alpha}\right) x+1\right) t}\left(\sum_{l=0}^{\infty} \frac{\left(-q^{\alpha}\right)^{l}}{1+q^{\alpha l}} \frac{t^{l}}{l !}\right) \\
& =[2]_{q}\left(\sum_{m=0}^{\infty}\left(\left(1-q^{\alpha}\right) x+1\right)^{m} \frac{t^{m}}{m !}\right)\left(\sum_{l=0}^{\infty} \frac{\left(-q^{\alpha}\right)^{l}}{1+q^{\alpha l}} \frac{t^{l}}{l !}\right) \\
& =[2]_{q} \sum_{n=0}^{\infty} \sum_{l=0}^{n}\left(\begin{array}{l}
n \\
l
\end{array}\right) \frac{\left(\left(1-q^{\alpha}\right) x+1\right)^{n-l}}{1+q^{\alpha l}}\left(-q^{\alpha}\right)^{l} \frac{t^{n}}{n !},
\end{aligned}
$$

by (11) and (12), we get

$$
\begin{aligned}
\left(1-q^{\alpha}\right)^{n} \tilde{\epsilon}_{n, q}^{(\alpha)}(x) & =[2]_{q} \sum_{l=0}^{n}\left(\begin{array}{l}
n \\
k
\end{array}\right) \frac{\left(\left(1-q^{\alpha}\right) x+1\right)^{n-l}}{1+q^{\alpha l}}\left(-q^{\alpha}\right)^{l} \\
& =[2]_{q} \sum_{l=0}^{n}\left(\begin{array}{l}
n \\
l
\end{array}\right) \frac{\left(-q^{\alpha}\right)^{l}}{1+q^{\alpha l}} \sum_{j=0}^{n-l}\left(\begin{array}{c}
n-l \\
j
\end{array}\right)\left(1-q^{\alpha}\right)^{j} x^{j}
\end{aligned}
$$

Thus, we have the following result.

Theorem 2.1 For $n \geq 1$,

$$
\begin{aligned}
\tilde{\epsilon}_{n, q}^{(\alpha)}(x) & =\frac{[2]_{q}}{\left(1-q^{\alpha}\right)^{n}} \sum_{l=0}^{n}\left(\begin{array}{l}
n \\
l
\end{array}\right) \frac{\left(-q^{\alpha}\right)^{l}\left(\left(1-q^{\alpha}\right) x+1\right)^{n-l}}{1+q^{\alpha l}} \\
& =\frac{[2]_{q}}{\left(1-q^{\alpha}\right)^{n}} \sum_{l=0}^{n} \sum_{j=0}^{n-l}\left(\begin{array}{c}
n \\
l
\end{array}\right)\left(\begin{array}{c}
n-l \\
j
\end{array}\right) \frac{\left(-q^{\alpha}\right)^{l}\left(1-q^{\alpha}\right)^{j}}{1+q^{\alpha l}} x^{j} .
\end{aligned}
$$

A systemic study of some families of the modified $q$-Euler polynomials with weight is presented by using the multivariate fermionic $p$-adic integral on $\mathbb{Z}_{p}$. The study of these 
modified $q$-Euler numbers and polynomials yields an interesting $q$-analogue of identities for Stirling numbers.

In recent years, many mathematicians and physicists have investigated zeta functions, multiple zeta functions, $L$-functions, and multiple $q$-Bernoulli numbers and polynomials mainly because of their interest and importance. These functions and polynomials are used not only in complex analysis and mathematical physics, but also in $p$-adic analysis and other areas. In particular, multiple zeta functions and multiple $L$-functions occur within the context of knot theory, quantum field theory, applied analysis and number theory (see [1-29]).

In our subsequent papers, we shall apply this $p$-adic mathematical theory to quantum statistical mechanics. Using $p$-adic quantum statistical mechanics, we can also derive a new partition function in the $p$-adic space and adopt this new partition function to quantum transport theory which is based on the projection technique related to the Liouville equation. We expect that a new quantum transport theory will explain diverse physical properties of the condensed matter system.

Competing interests

The authors declare that they have no competing interests.

Authors' contributions

All authors contributed equally to the manuscript and typed, read, and approved the final manuscript.

\section{Author details}

'Department of Mathematics Education, Kyungpook National University, Taegu, 702-701, Republic of Korea.

${ }^{2}$ Department of Mathematics, Kyungpook National University, Taegu, 702-701, Republic of Korea.

\section{Acknowledgements}

The authors are grateful for the valuable comments and suggestions of the referees.

Received: 23 September 2013 Accepted: 19 November 2013 Published: 05 Dec 2013

References

1. Araci, S, Acikgoz, M: A note on the Frobenius-Euler numbers and polynomials associated with Bernstein polynomials. Adv. Stud. Contemp. Math. 22(3), 399-406 (2012)

2. Araci, S, Acikgoz, M, Şen, E: On the extended Kim's $p$-adic $q$-deformed fermionic integrals in the $p$-adic integer ring. J. Number Theory 133, 3348-3361 (2013)

3. Araci, S, Acikgoz, M, Jolany, H: On p-adic interpolating function associated with modified Dirichlet's type of twisted $q$-Euler numbers and polynomials with weight. J. Class. Anal. 1(1), 35-48 (2013)

4. Bayad, A: Special values of Lerch zeta function and their Fourier expansions. Adv. Stud. Contemp. Math. 21(1), 1-4 (2011)

5. Carlitz, L: q-Bernoulli numbers and polynomials. Duke Math. J. 15, 987-1000 (1948)

6. Can, M, Cenkci, M, Kurt, V, Simsek, Y: Twisted Dedekind type sums associated with Barnes' type multiple Frobenius-Euler l-functions. Adv. Stud. Contemp. Math. 18(2), 135-160 (2009)

7. Cenkci, M, Simsek, Y, Kurt, V: Multiple two-variable $p$-adic $q$ - $L$-function and its behavior at $s=0$. Russ. J. Math. Phys. 15(4), 447-459 (2008)

8. Choi, J, Kim, T, Kim, YH: A note on the modified $q$-Euler numbers and polynomials with weight. Proc. Jangjeon Math. Soc. 14(4), 399-402 (2011)

9. Choi, J, Kim, T, Kim, YH, Lee, B: On the (w, q)-Euler numbers and polynomials with weight $\alpha$. Proc. Jangjeon Math. Soc. 15(1), 91-100 (2012)

10. Ding, D, Yang, J: Some identities related to the Apostol-Euler and Apostol-Bernoulli polynomials. Adv. Stud. Contemp. Math. 20(1), 7-21 (2010)

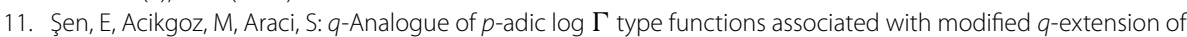
Genocchi numbers withe weight $\alpha$ and $\beta$. Turk. J. Math. Anal. Number Theory 1, 9-12 (2013). doi:10.12691/tjant-1-1-3

12. Kim, DS: Identities associated with generalized twisted Euler polynomials twisted by ramified roots of unity. Adv. Stud. Contemp. Math. 22(3), 363-377 (2012)

13. Kim, DS, Lee, N, Na, J, Pak, HK: Identities of symmetry for higher-order Euler polynomials in three variables (I). Adv. Stud. Contemp. Math. 22(1), 51-74 (2012)

14. Kim, DS, Kim, T, Kim, YH, Lee, SH: Some arithmetic properties of Bernoulli and Euler numbers. Adv. Stud. Contemp. Math. 22(4), 467-480 (2010)

15. Kim, T: Some identities for the Bernoulli, the Euler and the Genocchi numbers and polynomials. Adv. Stud. Contemp. Math. 20(1), 23-28 (2010) 
16. Kim, T: On the weighted $q$-Bernoulli numbers and polynomials. Adv. Stud. Contemp. Math. 21(2), 207-215 (2011)

17. Kim, T: Identities on the weighted $q$-Euler numbers and $q$-Bernstein polynomials. Adv. Stud. Contemp. Math. 22(1), 7-12 (2012)

18. Kim, T: An identity of the symmetry for the Frobenius-Euler polynomials associated with the fermionic $p$-adic invariant $q$-integrals on $\mathbb{Z}_{p}$. Rocky Mt. J. Math. 41(1), 239-247 (2011)

19. Kim, T: Some identities on the $q$-Euler polynomials of higher order and $q$-Stirling numbers by the fermionic $p$-adic integral on $\mathbb{Z}_{p}$. Russ. J. Math. Phys. 16(4), 484-491 (2009)

20. Kim, T: q-Generalized Euler numbers and polynomials. Russ. J. Math. Phys. 13(3), 293-298 (2006)

21. Kim, T: $q$-Volkenborn integration. Russ. J. Math. Phys. 9(3), 288-299 (2002)

22. Kim, T: The modified $q$-Euler numbers and polynomials. Adv. Stud. Contemp. Math. 16(2), 161-170 (2008)

23. Rim, SH, Jeong, J: On the modified q-Euler numbers of higher order with weight. Adv. Stud. Contemp. Math. 22(1), 93-98 (2012)

24. Rim, SH, Jeong, J: A note on the modified $q$-Euler numbers and polynomials with weight $\alpha$. Int. Math. Forum 6(56), 3245-3250 (2011)

25. Kim, T: Symmetry of power sum polynomials and multivariated fermionic $p$-adic invariant integral on $\mathbb{Z}_{p}$. Russ. J. Math. Phys. 16(1), 93-96 (2009)

26. Kim, T: Identities involving Frobenius-Euler polynomials arising from non-linear differential equations. J. Number Theory 132(12), 2854-2865 (2012)

27. Kim, T, Choi, JY, Sug, JY: Extended $q$-Euler numbers and polynomials associated with fermionic $p$-adic $q$-integral on $\mathbb{Z}_{p}$. Russ. J. Math. Phys. 14(2), 160-163 (2007)

28. Kim, T, Kim, DS, Bayad, A, Rim, SH: Identities on the Bernoulli and the Euler numbers and polynomials. Ars Comb. 107, 455-463 (2012)

29. Kim, T, Kim, DS, Dolgy, DV, Rim, SH: Some identities on the Euler numbers arising from Euler basis polynomials. Ars Comb. 109, 433-446 (2013)

10.1186/1687-1847-2013-356

Cite this article as: Rim et al.: On the modified $q$-Euler polynomials with weight. Advances in Difference Equations 2013, 2013:356

\section{Submit your manuscript to a SpringerOpen ${ }^{\circ}$ journal and benefit from:}

- Convenient online submission

Rigorous peer review

- Immediate publication on acceptance

- Open access: articles freely available online

- High visibility within the field

- Retaining the copyright to your article 